\title{
Bragg spectroscopy of discrete axial quasiparticle modes in a cigar-shaped degenerate Bose gas
}

\author{
Tarun Kanti Ghosh \\ The Abdus Salam International Center for Theoretical Physics, Strada Costiera 11, 34014 Trieste, Italy.
}

(Dated: November 14, 2018)

\begin{abstract}
We propose an experiment in which long wavelength discrete axial quasiparticle modes can be imprinted in a 3D cigar-shaped Bose-Einstein condensate by using two-photon Bragg scattering experiments, similar to the experiment at the Weizmann Institute [J. Steinhauer et al., Phys. Rev. Lett. 90, 060404 (2003)] where short wavelength axial phonons with different number of radial modes have been observed. We provide values of the momentum, energy and time duration of the two-photon Bragg pulse and also the two-body interaction strength which are needed in the Bragg scattering experiments in order to observe the long wavelength discrete axial modes. These discrete axial modes can be observed when the system is dilute and the time duration of the Bragg pulse is long enough.

PACS numbers: PACS numbers: 03.75.Kk, 32.80.Lg, 67.40.Db
\end{abstract}

Bose-Einstein condensates (BEC) [1] of alkali-atoms represent a wonderful testing ground of theories of weakly interacting bosons. The atoms are spatially confined by a harmonic trap and inhomogeneity makes these systems even more interesting. By changing the trapping frequency one can produce quasi-1D, quasi-2D as well as cigar-shaped but 3D Bose systems. Due to the reduction in the dimension and different shape, the effect of density and phase fluctuations become prominent which produces many interesting features. Bragg spectroscopy [2, 3] of a trapped BEC has become an important tool to reveal many bulk properties such as dynamic structure factor, verification of the Bogoliubov excitation spectrum [4, 5], observation of the Bogoliubov quasiparticle amplitudes [6], momentum distribution and correlation functions of a phase fluctuating quasi-1D Bose systems [7, 8]. Bragg spectroscopy has also been used for tuning and measuring the scattering length of atoms by using the optical Feshbach resonance [9].

In the Bragg scattering experiments, the condensate is excited by using two Bragg pulses with approximately parallel polarization, separated by an angle $\theta$. The pulses have a frequency difference $\omega$ determined by two acoustooptic modulators. If a photon is absorbed from the higher-frequency $\left(\omega_{h}\right)$ beam and emitted into other $\left(\omega_{l}\right)$, an excitation is produced with energy $\hbar \omega=\hbar\left(\omega_{h}-\omega_{l}\right)$ and momentum $\hbar \mathbf{k}$, where $k=|\mathbf{k}|=2 k_{p} \sin (\theta / 2)$, and $k_{p}$ is the photon wave number. The wave-vector $\mathbf{k}$ is adjusted to be along the $z$-axis. Both the values of $k$ and $\omega$ can be tuned by changing the angle between two beams and varying their frequency difference. For small values of $k$, the system is excited in the phonon regime and the response is detected by measuring the net momentum imparted to the system. Note that, all the momentum response experiments [3, 4, 5] are limited to at most a quarter of radial trapping period $T_{0}\left(T_{0}=2 \pi / \omega_{0}\right)$ and the energy transfer $\omega$ is chosen to be the order of $\mathrm{kHz}$ so that $\omega>\omega_{0}$ (where $\omega_{0}$ is the radial trapping frequency) which excites the different radial modes. In those experiments 3, 4], the local density approximation has been used to the actual inhomogeneous condensates, by using the Bogoliubov theory of uniform gases.

The axial excitations of a cigar shaped BEC can be divided into two regimes: short wavelength excitations whose wavelength is much smaller than the axial size and long wavelength excitations whose wavelength is equal or larger than the axial size of the system. The short wavelength axial phonons with different number of radial modes of a cigar-shaped condensates which give rise to the multibranch spectrum [10] has been resolved in a Bragg spectroscopy with a long duration $\left(t_{B}>T_{0}\right)$ of the Bragg pulses [5, 11]. In this experiment the condensate is excited by the Bragg pulses and allow it for free expansion. During the expansion of the condensate, these short wavelength excitations decouple from the condensate itself, forming a separate cloud of atoms. The total momentum is measured by counting the atoms which is decoupled from the condensate.

We consider a 3D Bose gas confined in a cigar-shaped harmonic trap. As described in Ref. [5], the condensate consists of $N=10^{5}$ atoms of ${ }^{87} \mathrm{Rb}$. The radial and axial trapping frequencies are $\omega_{0}=2 \pi \times 220 \mathrm{~Hz}$ and $\omega_{z}=2 \pi \times 25 \mathrm{~Hz}\left(a_{z}=\sqrt{\hbar / m \omega_{z}}=2.155 \mu m\right)$, respectively. Recently, the low-energy axial modes of a 3D BEC has been studied theoretically [13] and experimentally 14 by using a matter-wave interference technique. In those works 13, 14], they have shown that the long wavelength phonon do not form a separate cloud during the expansion. At long expansion times and for a given $k$, the excitations can separate out from the condensate only if $k a_{0}>k_{c} a_{0}=\pi\left(\omega_{z} / \omega_{0}\right)(\eta / 2)^{1 / 2}$, while for $k<k_{c}$, they remain within the condensate at all times [13]. Here, $\eta=\mu / \hbar \omega_{0}$ and $\mu$ is the chemical potential. Therefore, in order to observe the long wavelength discrete axial modes by using the Bragg spectroscopy as in Ref. [5], we have to choose the parameters such that the 
low energy excitations can separate out from the condensate for low value of $k$. This is an alternative way to realize the low-energy discrete axial modes in contrast to the matter-wave interference technique [14].

In order to get the separate cloud of low-energy axial modes, the chemical potential $\eta$ must be small. One can reduce the two-body scattering length $a$ by using the Feshbach resonance [9] so that the axial size decreases and the long wavelength excitations can separate out easily from the condensate. If we take $a=13.63 \times 10^{-11} \mathrm{~m}$, instead of the bare scattering length for Rubidium and other parameters are remain fixed as it is in Ref. [5], then $\mu=2 \hbar \omega_{0}$. Of course, one can also get the chemical potential $\mu=2 \hbar \omega_{0}$ by reducing the number of atoms in the condensate while keeping the bare value of the twobody scattering length. For this choice of $a, k_{c} a_{0}=0.35$ and we will show that the long wavelength excitations can be separated out of the condensate. Note that for this choice of scattering length, the system is still being 3D. The radial and axial sizes of the condensate are $R_{0} \sim 1.46 \mu \mathrm{m}$ and $Z_{0} \sim 12.78 \mu \mathrm{m}$, respectively, so that it becomes a cigar-shaped condensate with the deformation parameter $\lambda=\omega_{0} / \omega_{z} \sim 9$.

Note that in the experiment [ $\underline{5}$, the energy transfer $\omega$ is chosen to be the order of $\mathrm{kHz}\left(\omega>\omega_{0}\right)$ which excites the short wavelength axial phonons with different radial modes. Since the wave-lengths of these excitations are very small compared to the axial size and large compared to the radial size, then the axial quasiparticle modes can be described by the plane-wave states and the radial excitations can be described by the discrete modes which are the manifestation of the inhomogeneity along the radial direction. Therefore, the short wavelength phonons propagate along the $z$-axis with different number of radial modes which have been observed in Ref. [5]. If $\omega$ is the order of $\omega_{z}$ but less than the radial trapping frequency $\omega_{0}$ i.e. $\omega_{z} \leq \omega<<\omega_{0}$, and the wave-vector of the Bragg pulse is comparable to the inverse of the axial size then it would excite only the different number of long wavelength axial modes, instead of short wavelength axial phonons with different numbers of radial modes. Therefore, the wave-length of these excitations are comparable to the axial size and the effect of inhomogeneity along the axial direction has to be included in the studies. The inhomogeneity along the axial direction would be manifested by the presence of the discrete axial modes. The discrete long wavelength axial modes due to the finite size of the axial direction can be observed by measuring the dynamic structure factor, $S(k, \omega)$, which is related to the momentum transferred $P_{z}(t)$ due to the low-energy two-photon Bragg scattering.

Within the Thomas-Fermi approximation (i. e. neglecting the quantum pressure term), the hydrodynamic description for the axial density fluctuations $\delta n(z)$ of a $3 \mathrm{D}$ cigar shaped Bose system has been studied in [15]. In the dimensionless form, the equation for the $\delta n(z)$ reads as

$$
\left[\left(1-\tilde{z}^{2}\right) \nabla_{\tilde{z}}^{2}-4 \tilde{z} \nabla_{\tilde{z}}+4 \tilde{\omega}^{2}\right] \delta n(\tilde{z})=0,
$$

where $\tilde{z}=z / Z_{0}$ and $\tilde{\omega}=\omega / \omega_{z}$. The eigenvalues of the above equation are $\left(\omega_{j} / \omega_{z}\right)^{2}=j(j+3) / 4$ and the corresponding normalized eigen functions are

$$
\delta n(\tilde{z}) \sim \psi_{j}(\tilde{z})=\sqrt{\frac{(j+2)(2 j+3)}{8(j+1) \pi R_{0}^{2} Z_{0}}} P_{j}^{(1,1)}(\tilde{z}),
$$

where $P_{j}^{(1,1)}(\tilde{z})$ is the Jacobi polynomial. Eq. (10), including the above eigenvalues and the eigen vectors are valid only when the system parameter satisfies the condition: $\mu>>\hbar \omega_{0}>>\hbar \omega_{z}$. In our method, the chemical potential is not too large compared to the first radial excitation, but it is quite large compared to the first axial excitation. Therefore, the Thomas-Fermi approximation is a reasonable approximation, since we are dealing with the axial modes only and there is no coupling with the radial modes. The quantum numbers $j=1$ and $j=2$ correspond to the axial center-of-mass and the breathing modes with the frequencies $\omega_{1}=\omega_{z}$ and $\omega_{2}=\sqrt{5 / 2} \omega_{z}$, respectively. Only these two frequencies have been measured with high accuracy by using the time-dependent modulation of the trapping potential 16. It is very difficult to measure the frequencies of the other axial modes of large quantum numbers by using the time-dependent modulation of the trapping potential and therefore there are no measurements of the frequencies of these higher modes. It is useful to verify the frequencies of the other axial modes $(j>2)$ to make sure that the energy eigen values derived from the hydrodynamic approximation are correct. These modes can be easily verified by using the Bragg spectroscopy which is also our main concern of this work.

The dynamic structure factor is obtained from the Fourier transform of the time-dependent density-density correlation functions,

$$
S(k, \omega)=\int d t \int d z e^{i(\omega t-k z)}<\delta \hat{n}(z, t) \delta \hat{n}(0,0)>,
$$

where the density fluctuation operator is

$$
\delta \hat{n}(\tilde{z}, t)=\sum_{j} i \sqrt{\frac{\hbar \omega_{j}}{2 g}} \psi_{j}(\tilde{z}) e^{-i \omega_{j} t} \hat{\alpha}_{j}+\text { H.c. }
$$

Here, $g=4 \pi a \hbar^{2} / m$ is the interaction strength determined by the $s$-wave scattering length $a$ and $\hat{\alpha}_{j}$ is the quasi-particle operator of the $j$-th mode. It is the density fluctuation spectrum that can be measured in the two-photon Bragg spectroscopy. At $T=0$, the dynamic structure factor can be written as

$$
S(k, \omega)=\sum_{j} A_{j}\left|\psi_{j}(k)\right|^{2} \delta\left(\omega-\omega_{j}\right),
$$


where $A_{j}=\left(\frac{R_{0}^{2} Z_{0}}{128 \pi^{2} a a_{z}^{2}}\right) \frac{\sqrt{j(j+3)}(j+2)(2 j+3)}{(j+1)}$ and $\psi_{j}(\tilde{k})=$ $\int_{-1}^{1} d \tilde{z} e^{-i \tilde{k} \tilde{z}} P_{j}^{(1,1)}(\tilde{z})$ is the spatial Fourier transform of $P_{j}^{(1,1)}(\tilde{z})$. Here, $\tilde{k}=k Z_{0}$ is the dimensionless wave vector. We rewrite the dynamic structure factor as $S(\tilde{k}, \omega)=\sum_{j} S_{j}(\tilde{k}) \delta\left(\omega-\omega_{j}\right)$, where $S_{j}(\tilde{k})=A_{j}\left|\psi_{j}(k)\right|^{2}$ is the weight factor which determines the weight of the Bragg-scattering cross-section in $S(k, \omega)$ of the corresponding axial modes of energy $\hbar \omega_{j}$. In Fig.1 we show $S_{j}(\tilde{k})$ as a function of the dimensionless wave vector $\tilde{k}=k Z_{0}$ for the excitations $j=1,2$, and 3. Fig.1 shows how many axial modes significantly contribute to $S(k, \omega)$ for a given momentum $\hbar k$. It is clear from Fig.1 that the strongest weights for these collective modes appear for $k Z_{0} \geq 2$. As an example, for our choice of parame-

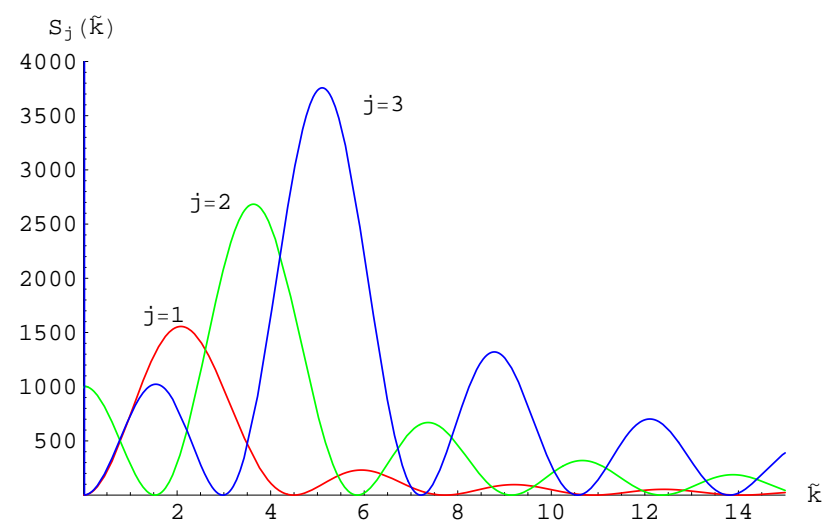

FIG. 1: (Color online) Plots of the weight factor $S_{j}(\tilde{k})$ vs the dimensionless wave-vector $\tilde{k}$ for $j=1,2$, and 3 .

ters which gives $Z_{0} \sim 5.932 a_{z}$, this means that the momentum transfer $\hbar k$ in a Bragg scattering experiments should be $k \geq 0.337 a_{z}^{-1}$ for $N=10^{5}$ in order to pick up the strong spectral weight from the low-energy collective modes. However, in order to get the separate cloud of the low-energy excited atoms from the condensate, the wave-vector of the Bragg pulse must be $k \geq 0.35 a_{0}^{-1}$ which implies that the wave vector of the Bragg pulse must satisfy the condition: $k Z_{0} \geq 6$.

We compare the dynamic structure factor given in Eq. (5D) with the dynamic structure factor calculated within the local-density approximation (LDA). In the LDA, the dynamic structure factor is given by the analytic expression 3, 17]

$S_{L D A}(\mathbf{k}, \omega)=\frac{15 \hbar^{2}}{8} \frac{\left(\hbar^{2} \omega^{2}-E_{r e}^{2}\right)}{E_{r e} \mu^{2}}\left[1-\frac{\left(\hbar^{2} \omega^{2}-E_{r e}^{2}\right)}{2 E_{r e} \mu}\right]^{\frac{1}{2}}$

Here, $E_{r e}=\frac{\hbar^{2} k^{2}}{2 m}$ is the recoil energy and $\mu=$ $0.5 \hbar \omega_{z}\left(15 \lambda^{2} N a / a_{z}\right)^{2 / 5}$ is the chemical potential. This analytic expression can also be used for 3D cigar-shaped trapped Bose systems. The above expression can be re- cast for this system as,

$S_{L D A}(\mathbf{k}, \omega)=\frac{15}{2 \omega_{z} C^{2}}\left[2\left(\frac{\tilde{\omega}}{\tilde{k}}\right)^{2}-\frac{\tilde{k}^{2}}{2 C^{4}}\right]\left[1-2\left(\frac{\tilde{\omega}}{\tilde{k}}\right)^{2}+\frac{\tilde{k}^{2}}{2 C^{4}}\right]^{\frac{1}{2}}$,

where $C=\left(15 \lambda^{2} N a / a_{z}\right)^{1 / 5} \sim 5.93$ for the experiment [5] and $\tilde{\omega}=\omega / \omega_{z}$. In Fig.2 we plot the dynamic structure factor $S(\tilde{k}, \omega)$ vs the frequency $\omega$ by using the LDA as well as by using the Fourier transformation of the densitydensity correlation function given in Eq. (5). For finiteenergy resolution we have replaced the delta function in Eq. (5) by the Lorentzian with a width of $\Gamma=0.1 \omega_{z}$.

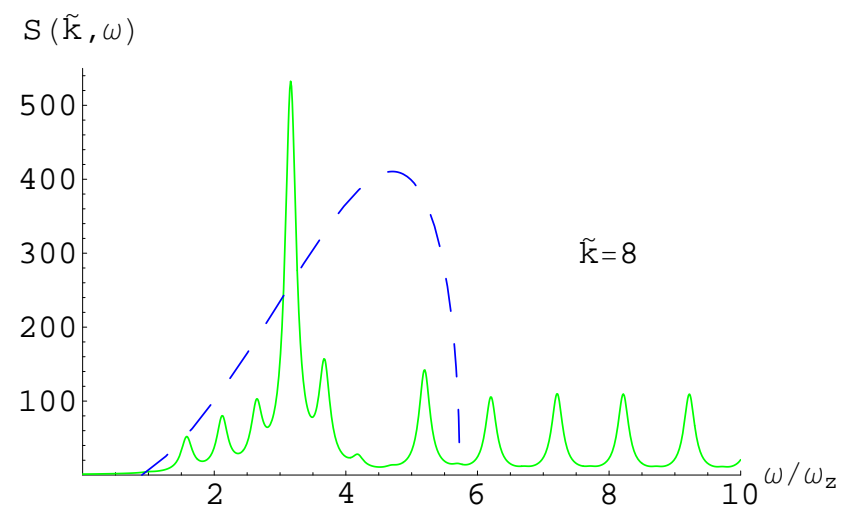

FIG. 2: (Color online) Plots of the dynamic structure factor $S(\tilde{k}, \omega)$ vs normalized frequency $\omega / \omega_{z}$ at $T=0$. The dashed lines shows the dynamic structure factor based on the LDA.

As one can see from Fig.2, the dynamic structure factor has multiple peaks. This phenomena is due to the underlying discrete axial spectrum. Fig. 2 shows that for the given $\tilde{k}=8$, the most prominent peaks at $\omega=3.162 \omega_{z}$ which corresponds to $j=5$ and other small peaks corresponds to the other quantum numbers. In order to observe the $j=5$ quasiparticle modes, the wave number of the Bragg pulse should be $\tilde{k}=8.0$ which can be obtained by suitable choices of the photon wave number $\left(k_{p}\right)$ and the angle $(\theta)$ between the Bragg beams. Note that, $\tilde{k}=8.0$ implies $k a_{0} \sim 0.45>k_{c} a_{0}$. Therefore, the excited states will be separated out from the condensate and the momentum measurements can be performed. The simplified LDA picture fails to produce the discrete structure of the axial modes, and it describes poorly the envelope of the spectrum of the dynamical structure factor. However, the single peak in $S_{L D A}$ occurs at $\omega_{m} \sim 4.75$ for $\tilde{k}=8$, but the locations of the highest peak in $S(k, \omega)$ is $\omega / \omega_{z}=3.162$. Therefore, the highest peak in $S(k, \omega)$ do not match with the peak in the $S_{L D A}(k, \omega)$. The LDA fails to describe the discrete structure of the modes because it assumes the system is locally uniform and it does consider partially the effect of the finite axial size. We have also carried out the same analysis for higher values of $k$. For $\tilde{k}=9$, the condensate 
responds resonantly at the frequency $\omega=3.674 \omega_{z}$ which corresponds to the axial quantum number $j=6$. In both the cases, the resonantly excited states are in the phonon regime since $k \xi<<1$, where $\xi=\sqrt{\hbar^{2} / 2 m \mu} \sim 0.168 a_{z}$ is the healing length.

The behavior of these multiple peaks in the dynamic structure factor can be resolved in two-photon Bragg spectroscopy, as shown by Steinhauer et al. [5]. In the two-photon Bragg spectroscopy, the dynamic structure factor can not be measured directly. Actually, the observable in the Bragg scattering experiments is the momentum transferred to the condensate which is related to the dynamic structure factor and reflects the behavior of the quasiparticle energy spectrum. The populations in the quasiparticle states can be controlled by using the two-photon Bragg pulse. When the condensate is irradiated by an external moving optical potential $V_{o p}=V_{B}(t) \cos (k z-\omega t)$, the excited states are populated by the quasiparticle with energy $\hbar \omega$ and the momentum $\hbar k$, depending on the value of $k$ and $\omega$ of the optical potential $V_{o p}$. Here, $V_{B}$ is the intensity of the Bragg pulse. Suppose the system is subjected to a timedependent Bragg pulse which is switched on at time $t>0$ and $k$ is also along the $z$-direction. The momentum transfer to the Bose system from the optical potential can be calculated analytically either by using the Bogoliubov transformation [18] or by using the phase-density representations of the bosonic order parameter [19] and it is given by

$$
\begin{aligned}
P_{z}(t) & =\sum_{j, k} \hbar k<\hat{\alpha}_{j}^{\dagger}(t) \hat{\alpha}_{j}(t)>=\left(\frac{V_{B}(t)}{2 \hbar}\right)^{2} \sum_{j} \hbar k S_{j}(\tilde{k}) \\
& \times F_{j}\left[\left(\omega_{j}-\omega\right), t\right]-F_{j}\left[\left(\omega_{j}+\omega\right), t\right],
\end{aligned}
$$

where $\hat{\alpha}_{j}(t)$ is the time-evolution of the quasiparticle operator of energy $\hbar \omega_{j}$ and $F_{j}\left[\left(\omega_{j} \pm \omega\right), t\right]=$ $\left(\frac{\sin \left[\left(\omega_{j} \pm \omega\right) t / 2\right]}{\left(\omega_{j} \pm \omega\right) / 2}\right)^{2}$. For positive $\omega$ and a given $\tilde{k}$ such that $S_{j}(\tilde{k})$ is maximum, the momentum transferred $P_{z}(t)$ is resonant at the frequencies $\omega=\omega_{j}$. The width of the each peak goes like $2 \pi / t$. In order to resolve the different peaks, the duration of the Bragg pulses should be at least of the order of the axial trapping period $T_{z}=2 \pi / \omega_{z}$. Moreover, the duration of the Bragg pulse, $t_{B}$, (atomlight interaction time) is a main factor in the Bragg spectroscopy and it must be of order $t_{B}>m / \hbar k^{2}$ in order to populate the Bragg reflected beam significantly [20]. For large $t$ and $\omega_{z}<<\omega_{0}, P_{z}(t) \sim S(k, \omega)$ 11]. In Fig.3, we plot the net momentum transfer $P_{z}(t)$ vs the frequency $\omega$ for three different choices of the time duration of the Bragg pulse. Fig. 3 shows that the shape of the $P_{z}(t)$ strongly depends on the time duration of the Bragg pulse $t_{B}$. When $t_{B}=1.1 T_{z}$, the $P_{z}(t)$ is a smooth curve with a single peak at $\omega / \omega_{z}=1.8$, where $T_{z}=2 \pi / \omega_{z}$ is the axial trapping period which is $40 \mathrm{msec}$ for the experiment $[\underline{5}$. When $t_{B}=1.9 T_{z}$, there is a little evidence of few small peaks start developing in the $P_{z}(t)$. When $t_{B}=3 T_{z}$, the multiple peaks in the $P_{z}(t)$ appears prominently. The location of the peaks in Fig.3 for $t_{B}=3 T_{z}$ are exactly same as in Fig.2. It implies that $P_{z}(t) \sim S((k, \omega)$ for a long duration of the Bragg pulse. Therefore, the multiple peaks in $S(k, \omega)$ are resolved in Fig.3 only when the duration of the Bragg pulse is $t_{B}>>T_{z}$. It should be noted that it is very difficult to use long duration of the Bragg pulse at the present situation. It can induce a dipole oscillation of the whole condensate in the trap [21] and the reflection of phonons at the boundaries which might causes a broadening of the response. We have checked that the $P_{z}(t)$ reflects the dynamic structure factor calculated from the local density approximation if $t_{B}<T_{z}$. We also found that Fig. 3 and the locations of the discrete peaks does not depend on the intensity $V_{B}$ of the optical potential. We have also studied $P_{z}(t)$ for $\tilde{k}=9$ and find

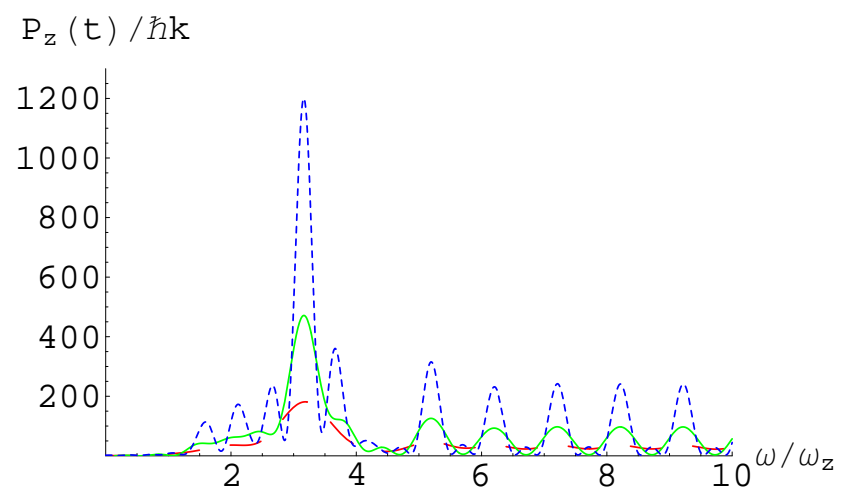

FIG. 3: (Color online) Plots of the net momentum transferred $P_{z}(t)$ vs normalized frequency $\omega / \omega_{z}$ at $T=0$ when the dimensionless wave-vector is $\tilde{k}=8$ for various time duration of the Bragg pulse: $t_{B}=1.1 T_{z}$ (dashed), $t_{B}=1.9 T_{z}$ (solid) and $t_{B}=3.0 T_{z}$ (dotted). Also, we have assumed $V_{B}=0.05 \hbar \omega_{z}$.

that there is a large peak at $\omega=3.674 \omega_{z}$ corresponds to the quantum number $j=6$ if the time duration of the Bragg pulse is longer than the axial trapping period. This peak is also occurred in the dynamic structure factor as we have already discussed.

One can do the same analysis for other values of $k$ as long as only the low-energy axial excited states contribute significantly in the dynamic structure factor and these low-energy axial states should be less than the first radial excitation. It would be interesting to study intermediate regimes of the energy and wave-vector where both the radial and axial discrete states are excited simultaneously and it would provide a rich physics of the dynamic structure factor as well as the momentum transfer to the Bose system, due to the non-trivial structure of spectrum of the density fluctuations and its corresponding eigenfunctions.

In conclusion, we have proposed an experiment in which the long wavelength discretized axial modes in a 
cigar-shaped (but 3D) condensate can be imprinted by using the Bragg scattering experiments, similar to the recent experiment 5] where the short wavelength axial phonons with discrete radial modes have been observed. We have estimated the two-body scattering length $a$, also the values of the momentum $(\hbar k)$ and energy $(\hbar \omega)$ of the two-photon Bragg pulses which are needed in the Bragg scattering experiments in order to observe the low energy discrete axial modes. These discrete axial modes can be observed when the system is dilute enough and the time duration of the Bragg pulse is long i.e. $t_{B}>>T_{z}$.

Present Address: Department of Physics, Okayama University, Okayama 700-8530, Japan. E-mail:tkghosh@mp.okayama-u.ac.jp

[1] F. Dalfovo, S. Giorgini, L. Pitaevskii, and S. Stringari, Rev. Mod. Phys. 71, 463 (1999).

[2] J. Stenger et al., Phys. Rev. Lett. 82, 4569 (1999).
[3] D. M. Stamper-Kurn et al., Phys. Rev. Lett. 83, 2876 (1999).

[4] J. Steinhauer et al., Phys. Rev. Lett. 88, 120407 (2002).

[5] J. Steinhauer et al., Phys. Rev. Lett. 90, 060404 (2003).

[6] A. Brunello et al., Phys. Rev. Lett. 85, 4422 (2000).

[7] S. Richard et al., Phys. Rev. Lett. 91, 010405 (2003).

[8] F. Gerbier et al., Phys. Rev. A 67, 051602 (2003).

[9] M. Theis et al., Phys. Rev. Lett. 93, 123001 (2004).

[10] E. Zaremba, Phys. Rev. A 57, 518 (1998).

[11] C. Tozzo and F. Dalfovo, New J. Phys. 5, 54 (2003).

[12] D. S. Petrov, G. V. Shlyapnikov, and J. T. M. Walraven, Phys. Rev. Lett. 87, 050404 (2001).

[13] C. Tozzo and F. Dalfovo, Phys. Rev. A 69, 053606 (2004).

[14] N. Katz et al., Phys. Rev. Lett. 93, 220403 (2004)

[15] M. Fliesser et al., Phys. Rev. A 56, R2533 (1997); S. Stringari, Phys. Rev. A 58, 2385 (1998).

[16] M.-O. Mewes et al., Phys. Rev. Lett. 77, 988 (1996).

[17] F. Zambelli et al., Phys. Rev. A 61, 063608 (2000).

[18] P. B. Blakie et al., Phys. Rev. A 65, 033602 (2002).

[19] T. K. Ghosh, cond-mat/0402079

[20] C. Keller et al., Appl. Phys. B 69, 303 (1999).

[21] A. Brunello et al., Phys. Rev. A 64, 063614 (2001). 